SISSA-3/2001/FM

\title{
One-point functions in integrable quantum field theory at finite temperature
}

\author{
G. Delfino円 \\ International School for Advanced Studies and Istituto Nazionale di Fisica Nucleare \\ 34014 Trieste, Italy
}

\begin{abstract}
We determine the form factor expansion of the one-point functions in integrable quantum field theory at finite temperature and find that it is simpler than previously conjectured. We show that no singularities are left in the final expression provided that the operator is local with respect to the particles and argue that the divergences arising in the non-local case are related to the absence of spontaneous symmetry breaking on the cylinder. As a specific application, we give the first terms of the low temperature expansion of the one-point functions for the Ising model in a magnetic field.
\end{abstract}

\footnotetext{
${ }^{1}$ E-mail: delfino@sissa.it
} 
Quantum field theory at finite temperature is a subject of both theoretical and experimental interest. In the euclidean formulation, it corresponds to work with an immaginary time compactified on a circle whose circumference $R$ coincides with the inverse temperature. The average of a generic product $X$ of local operators is given by

$$
\langle X\rangle_{R}=\frac{\operatorname{Tr} X e^{-R H}}{\operatorname{Tr} e^{-R H}},
$$

where $H$ is the Hamiltonian.

In two dimensions, a non-perturbative study of the properties of the finite temperature case should be achievable exploiting the results of integrable quantum field theory. In particular, a natural idea is that of approaching the computation of the correlation functions in a way similar to that successfully used in the zero temperature case, and evaluate (1) on the basis of the multi-particle asymptotic states of the zero temperature theory. At the end, the problem should be reduced to summing over the matrix elements of the operators between the vacuum and the $n$-particle asymptotic states (the form factors) which are known exactly in integrable theories.

The one-point functions have a non-trivial temperature dependence and provide the first test that this form factor method needs to pass. Following the analysis of the free fermionic case [1], an extension to the generic integrable case exploiting also the results of the thermodynamic Bethe ansatz (TBA) was conjectured in Ref. [2]. More precisely, it was proposed that the 'pseudoenergy' given by the TBA should substitute the ordinary energy of the particles in the thermal weight function entering the form factor sum. Lukyanov found agreement with this conjecture in his semi-classical study of the sinh-Gordon model at finite temperature [4].

In this note, we analyse explicitely the form factor expansion of the one-point functions in finite temperature integrable theories and find that the final expression, Eq. (16), is simpler than proposed in the past: the weight function is the same than in the free cases and all the effects of the interaction are contained in the form factors. Along the way we discuss a number of delicate issues originating from the fact that the expression (1) is a sum over matrix elements between identical asymptotic states which contain singularities. We explain why and in which cases the final result is free of divergences.

This analysis also allows a clarification of the status of the conjecture of Ref. [2]. In fact, the expression for the one-point functions proposed in that work differs from our Eq. (16) not only in the thermal weight, but also in the operator dependent part. In Refs. [2, 3], the latter does not coincide with the form factors (12) but is such that its combination with the modified weight function reproduces the TBA result for the one- 
point function of the trace of the stress-energy tensor. At present it is not clear how a similar expression involving the pseudoenergies could be derived for other operators. In any case, due to the complicated temperature dependence of the pseudoenergies, it would not be the explicit low temperature expansion (16).

Consider an integrable quantum field theory whose spectrum contains a single neutral particle of mass $M$. As usual, let us use the rapidity variable $\theta$ to parameterise the energy and momentum of a particle as $\left(p^{0}, p^{1}\right)=(M \cosh \theta, M \sinh \theta)$. Then relativistic invariant quantities depend on the rapidity differences only. We denote $S\left(\theta_{1}-\theta_{2}\right)$ the scattering amplitude of two particles with rapidities $\theta_{1}$ and $\theta_{2}$. It satisfies the crossing symmetry relation

$$
S(\theta)=S(i \pi-\theta)
$$

Integrability shows up in two respects [5]. First, it forbids any inelastic process, so that the unitarity condition takes the simple form

$$
S(\theta) S(-\theta)=1
$$

Second, it induces the complete factorisation of multi-particle scattering amplitudes into the product of the two-particle ones.

Take now a scalar operator $\Phi(x)$ in this theory which is local with respect to the particles, and denote its matrix elements on the asymptotic multi-particle states as

$$
F_{m, n}^{\Phi}\left(\theta_{m}^{\prime}, \ldots, \theta_{1}^{\prime} \mid \theta_{1}, \ldots, \theta_{n}\right)=\left\langle\theta_{m}^{\prime}, \ldots, \theta_{1}^{\prime}|\Phi(0)| \theta_{1}, \ldots, \theta_{n}\right\rangle
$$

The contribution of the $n$-particle asymptotic state to $\operatorname{Tr} \Phi(x) e^{-H R}$ is

$$
f_{n}^{\Phi}(R)=\frac{1}{n !} \frac{1}{(2 \pi)^{n}} \int d \theta_{1} \ldots d \theta_{n} F_{n, n}^{\Phi}\left(\theta_{n}, \ldots, \theta_{1} \mid \theta_{1}, \ldots, \theta_{n}\right) e^{-E_{n} R}
$$

where

$$
E_{n}=M \sum_{i=1}^{n} \cosh \theta_{i}
$$

is the total energy of the asymptotic state, and we used the property $\Phi(x)=e^{i P_{\mu} x^{\mu}} \Phi(0) e^{-i P_{\mu} x^{\mu}}$. $f_{n}^{\Phi}(R)$ is of order $e^{-n M R}$ for $M R \rightarrow \infty$. After defining

$$
f^{\Phi}(R)=\sum_{n=0}^{\infty} f_{n}^{\Phi}(R)
$$

we can write

$$
\langle\Phi\rangle_{R}=\frac{f^{\Phi}(R)}{f^{I}(R)},
$$


with $I$ denoting the identity operator.

It is our goal to rewrite this rather implicit expression containing the matrix elements $F_{n, n}^{\Phi}$ as a sum over the form factors $F_{0, n}^{\Phi}$ which are exactly computable in integrable field theories [6, 7]. Technically, this problem has several points of contact with Ref. [8], where non-integrable field theories were considered as perturbations of the integrable ones. In that context the matrix elements $F_{m, n}^{\Phi}$ determine the first order correction to the scattering process $m \rightarrow n$ under the action of the perturbing operator $\Phi$.

The reduction to the form factors $F_{0, n}^{\Phi}$ is performed iterating $m$ times the crossing relation [6, []

$$
\begin{aligned}
& F_{m, n}^{\Phi}\left(\theta_{m}^{\prime}, \ldots, \theta_{1}^{\prime} \mid \theta_{1}, \ldots, \theta_{n}\right)=F_{m-1, n+1}^{\Phi}\left(\theta_{m}^{\prime}, \ldots, \theta_{2}^{\prime} \mid \theta_{1}^{\prime}+i \pi, \theta_{1}, \ldots, \theta_{n}\right)+ \\
& 2 \pi \sum_{i=1}^{n} \delta\left(\theta_{1}^{\prime}-\theta_{i}\right) \prod_{k=1}^{i-1} S\left(\theta_{k}-\theta_{1}^{\prime}\right) F_{m-1, n-1}^{\Phi}\left(\theta_{m}^{\prime}, \ldots, \theta_{2}^{\prime} \mid \theta_{1}, \ldots, \theta_{i-1}, \theta_{i+1}, \ldots, \theta_{n}\right) .
\end{aligned}
$$

The second term in the r.h.s. accounts for the disconnected parts that appear if the crossed particle hits a particle with exactly the same momentum and annihilates it. It contains the product of the scattering amplitudes with the particles that need to be crossed on the way. We recall in fact that the relation

$$
\left|\ldots \theta_{i}, \theta_{i+1} \ldots\right\rangle=S\left(\theta_{i}-\theta_{i+1}\right)\left|\ldots \theta_{i+1}, \theta_{i} \ldots\right\rangle,
$$

is characteristic of integrable field theories. The situation of collinearity responsible for the disconnected parts also induces an 'annihilation' pole in the matrix elements with residue [7]

$$
-i \operatorname{res}_{\theta_{1}=\theta_{2}+i \pi} F_{0, n}^{\Phi}\left(\mid \theta_{1}, \ldots, \theta_{n}\right)=\left[1-\prod_{i=1}^{n} S\left(\theta_{i}-\theta_{2}\right)\right] F_{0, n-2}^{\Phi}\left(\mid \theta_{3}, \ldots, \theta_{n}\right) .
$$

Since (5) contains matrix elements involving two identical sets of momenta, it is obvious that we will have to care about both annihilation poles and singular disconnected parts containing $\delta(0)$ factors. We will see in a moment, however, that these potential sources of trouble drop out and leave a final result which is perfectly finite.

Using (9) the first few $f_{n}^{\Phi}(R)$ are found to be

$$
\begin{aligned}
f_{0}^{\Phi} & =\mathcal{F}_{0}^{\Phi}=\langle 0|\Phi| 0\rangle=\langle\Phi\rangle_{R=\infty}, \\
f_{1}^{\Phi}(R) & =\frac{1}{2 \pi} \int d \theta_{1} e^{-E_{1} R}\left[\mathcal{F}_{2}^{\Phi}+2 \pi \delta(0) \mathcal{F}_{0}^{\Phi}\right], \\
f_{2}^{\Phi}(R) & =\frac{1}{2} \frac{1}{(2 \pi)^{2}} \int d \theta_{1} d \theta_{2} e^{-E_{2} R}\left[\mathcal{F}_{4}^{\Phi}\left(\theta_{1}, \theta_{2}\right)+4 \pi\left(\delta\left(\theta_{1}-\theta_{2}\right) S(0)+\delta(0)\right) \mathcal{F}_{2}^{\Phi}\right. \\
& \left.+(2 \pi)^{2}\left(S(0) \delta^{2}\left(\theta_{1}-\theta_{2}\right)+\delta^{2}(0)\right) \mathcal{F}_{0}^{\Phi}\right],
\end{aligned}
$$




$$
\begin{aligned}
f_{3}^{\Phi}(R) & =\frac{1}{3 !} \frac{1}{(2 \pi)^{3}} \int d \theta_{1} d \theta_{2} d \theta_{3} e^{-E_{3} R}\left[\mathcal{F}_{6}^{\Phi}\left(\theta_{1}, \theta_{2}, \theta_{3}\right)+6 \pi\left(2 S(0) \delta\left(\theta_{1}-\theta_{3}\right)+\delta(0)\right) \mathcal{F}_{4}^{\Phi}\left(\theta_{1}, \theta_{2}\right)\right. \\
& +3(2 \pi)^{2}\left(2 \delta\left(\theta_{1}-\theta_{2}\right) \delta\left(\theta_{1}-\theta_{3}\right)+S(0) \delta^{2}\left(\theta_{1}-\theta_{2}\right)+2 S(0) \delta(0) \delta\left(\theta_{1}-\theta_{2}\right)+\delta^{2}(0)\right) \mathcal{F}_{2}^{\Phi} \\
& \left.+(2 \pi)^{3}\left(2 \delta\left(\theta_{1}-\theta_{2}\right) \delta\left(\theta_{1}-\theta_{3}\right) \delta\left(\theta_{2}-\theta_{3}\right)+3 S(0) \delta(0) \delta^{2}\left(\theta_{1}-\theta_{2}\right)+\delta^{3}(0)\right) \mathcal{F}_{0}^{\Phi}\right]
\end{aligned}
$$

where we introduced the notation

$$
\mathcal{F}_{2 n}^{\Phi}\left(\theta_{1}, \ldots, \theta_{n}\right)=\lim _{\epsilon \rightarrow 0} F_{0,2 n}^{\Phi}\left(\mid \theta_{n}+i \pi+\epsilon, \ldots, \theta_{1}+i \pi+\epsilon, \theta_{1}, \ldots, \theta_{n}\right)
$$

$\mathcal{F}_{2}^{\Phi}\left(\theta_{1}\right)$ is a constant by relativistic invariance. We inserted in Eq. (12) an infinitesimal displacent from the pole configurations and will discuss later the result of the limit. For the time being we stress that the displacement has to be the same for all rapidities. Any different choice would introduce an unjustified asymmetry among the particles.

Notice that, as a combined effect of the delta functions associated with the disconnected parts, the $S$-matrix appears explicitely in the expressions for the $f_{n}^{\Phi}$ only through the constant factor $S(0)$. It follows from Eq. (3) that

$$
S(0)= \pm 1
$$

Eq. (10) shows that $S(0)$ determines whether the statistics of the particles is bosonic or fermionic. This means that the expansion of $\langle\Phi\rangle_{R}$ in the generic integrable case is formally the same than in the free bosonic and fermionic cases, all the difference being encoded in the explicit expressions of the form factors.

The sum of the $f_{n}^{\Phi}$ we determined gives

$$
\begin{aligned}
f^{\Phi}(R) & =Z\left\{\mathcal{F}_{0}^{\Phi}+\frac{1}{2 \pi} \int d \theta_{1} e^{-E_{1} R}\left[1+S(0) e^{-E_{1} R}+e^{-2 E_{1} R}\right] \mathcal{F}_{2}^{\Phi}\right. \\
& +\frac{1}{2} \frac{1}{(2 \pi)^{2}} \int d \theta_{1} d \theta_{2} e^{-E_{2} R}\left[1+\left(e^{-M R \cosh \theta_{1}}+e^{-M R \cosh \theta_{2}}\right) S(0)\right] \mathcal{F}_{4}^{\Phi}\left(\theta_{1}, \theta_{2}\right) \\
& \left.+\frac{1}{3 !} \frac{1}{(2 \pi)^{3}} \int d \theta_{1} d \theta_{2} d \theta_{3} e^{-E_{3} R} \mathcal{F}_{6}^{\Phi}\left(\theta_{1}, \theta_{2}, \theta_{3}\right)+O\left(e^{-4 M R}\right)\right\}
\end{aligned}
$$

where all the singular delta function terms factorise into the quantity

$$
\begin{aligned}
Z & =1+\int d \theta_{1} e^{-E_{1} R} \delta(0)+\frac{1}{2} \int d \theta_{1} d \theta_{2} e^{-E_{2} R}\left[S(0) \delta^{2}\left(\theta_{1}-\theta_{2}\right)+\delta^{2}(0)\right] \\
& +\frac{1}{3 !} \int d \theta_{1} d \theta_{2} d \theta_{3} e^{-E_{3} R}\left[2 \delta\left(\theta_{1}-\theta_{2}\right) \delta\left(\theta_{1}-\theta_{3}\right) \delta\left(\theta_{2}-\theta_{3}\right)+3 S(0) \delta(0) \delta^{2}\left(\theta_{1}-\theta_{2}\right)+\delta^{3}(0)\right] \\
& +O\left(e^{-4 M R}\right) .
\end{aligned}
$$

Since $\mathcal{F}_{2 n}^{I}=\delta_{n, 0}$, we have $f^{I}=Z$, so that the singular disconnected parts completely cancel out in the ratio (8) without the need of any specific regularisation. 
One can continue the computation and work out the next contributions with $n>3$ to convince himself that the complete result is

$$
\langle\Phi\rangle_{R}=\sum_{n=0}^{\infty} \frac{1}{n !} \frac{1}{(2 \pi)^{n}} \int\left[\prod_{i=1}^{n} d \theta_{i} g\left(\theta_{i}, R\right) e^{-M R \cosh \theta_{i}}\right] \mathcal{F}_{2 n}^{\Phi}\left(\theta_{1}, \ldots, \theta_{n}\right),
$$

with

$$
g(\theta, R)=\frac{1}{1-S(0) e^{-M R \cosh \theta}} .
$$

This expression provides the explicit form factor expansion for the one-point function. It only remains to check that the specific form factor configuration (12) is not singular due to the annihilation poles. For this purpose, take the form factor $F_{0,2 n}^{\Phi}\left(\mid \theta_{1}, \ldots, \theta_{2 n}\right)$ and bring the first two particles on the singular configuration $\theta_{1} \rightarrow \theta_{2}+i \pi$. This pinching produces a pole with the residue given by Eq. (11). We then repeat the operation on the second pair of particles by taking $\theta_{3} \rightarrow \theta_{4}+i \pi$, and so on with all the $n$ pairs. This procedure produces $n$ poles. The numerator will contain the product

$$
\prod_{k=1}^{n}\left[1-\prod_{i=2 k+1}^{2 n} S\left(\theta_{i}-\theta_{2 k}\right)\right]
$$

where each string of scattering amplitudes inside the parenthesis is made of factors $S\left(\theta_{2 j}+\right.$ $\left.i \pi-\theta_{2 k}\right) S\left(\theta_{2 j}-\theta_{2 k}\right)$. It follows from (2) and (3) that such factors are equal to 1 , so that (18) gives $n$ zeros which cancel the poles and leave a finite result. We made these considerations on a form factor which differs from (12) only for the ordering of the particles, namely (recall Eq. (10) ) for an overall phase which does not affect the final conclusion.

For later convenience, we write down more explicitely the first few terms in the low temperature expansion. They read

$$
\begin{aligned}
\langle\Phi\rangle_{R} & =\mathcal{F}_{0}^{\Phi}+\frac{1}{\pi}\left[K_{0}(r)+S(0) K_{0}(2 r)\right] \mathcal{F}_{2}^{\Phi}+\frac{1}{\pi^{2}} \int_{0}^{\infty} d \theta K_{0}(2 r \cosh \theta) \mathcal{F}_{4}^{\Phi}(2 \theta, 0) \\
& +O\left(e^{-3 r}\right)
\end{aligned}
$$

with

$$
r \equiv M R
$$

For the specific case in which the operator $\Phi(x)$ coincides with the trace $\Theta(x)$ of the stress-energy tensor, an alternative and effective way of computing the one-point function is provided by the thermodynamic Bethe ansatz approach [9]. It is then interesting to make a check of the agreement of the two methods in this case. The one-point function 
of the trace is related to the ground state energy on the cylinder (without the bulk term) $E(r)$ as

$$
\langle\Theta\rangle_{R}=\langle\Theta\rangle_{R=\infty}+2 \pi \frac{M}{r} \frac{d}{d r}[r E(r)] .
$$

The TBA allows the determination of $E(r)$ from the knowledge of the $S$-matrix in the form

$$
\begin{gathered}
E(r)=-M \int \frac{d \theta}{2 \pi} L(\theta) \cosh \theta, \\
L(\theta) \equiv-S(0) \ln \left(1-S(0) e^{-\varepsilon(\theta)}\right),
\end{gathered}
$$

where the 'pseudoenergy' $\varepsilon(\theta)$ is determined by the integral equation

$$
\begin{gathered}
\varepsilon(\theta)=r \cosh \theta-\frac{1}{2 \pi} \int d \theta^{\prime} \varphi\left(\theta-\theta^{\prime}\right) L\left(\theta^{\prime}\right), \\
\varphi(\theta) \equiv-i \frac{d}{d \theta} \ln S(\theta) .
\end{gathered}
$$

The pseudoenergy $\varepsilon(\theta)$ tends to $r \cosh \theta$ in the zero temperature limit. Iterating (24) once with this initial condition and substituting into Eq. (22) produces the result?

$$
\frac{E(r)}{M}=-\frac{1}{\pi}\left[K_{1}(r)+S(0) \frac{1}{2} K_{1}(2 r)\right]-\frac{2}{\pi^{2}} \int_{0}^{\infty} d \theta K_{1}(2 r \cosh \theta) \varphi(2 \theta) \cosh \theta+O\left(e^{-3 r}\right) .
$$

We can now evaluate $\langle\Theta\rangle_{R}$ to this order from (21) using the identity $\frac{d}{z d z}\left[z K_{1}(z)\right]=$ $-K_{0}(z)$. The final result exactly coincides with (19) because it was shown in Ref. [8] (Eqs. (3.23), (3.24)) on completely general and independent basis that

$$
\begin{aligned}
& \mathcal{F}_{2}^{\Theta}=2 \pi M^{2} \\
& \mathcal{F}_{4}^{\Theta}\left(\theta_{1}, \theta_{2}\right)=8 \pi M^{2} \varphi\left(\theta_{1}-\theta_{2}\right) \cosh ^{2} \frac{\theta_{1}-\theta_{2}}{2} .
\end{aligned}
$$

We also recall here that nearby a fixed point the ground state energy behaves as 10 $E(R) \simeq-\pi C_{e f f} / 6 R$, where

$$
C_{e f f}=C-\frac{X_{\min }}{12}
$$

is given in terms of the central charge $C$ and smallest scaling dimension $X_{\text {min }}$ at the conformal point $\left(X_{\min }=0\right.$ in a 'unitary' theory). Integration of Eq. (21) then gives

$$
C_{e f f}^{U V}-C_{e f f}^{I R}=\frac{3}{\pi^{2} M^{2}} \int_{0}^{\infty} d r r\left(\langle\Theta\rangle_{R}-\langle\Theta\rangle_{R=\infty}\right)
$$

\footnotetext{
${ }^{1}$ In the computation one uses the fact that $\varphi(\theta)$ is an even function.

${ }^{2}$ It has been checked for many models that only the $\epsilon$-prescription (12) reproduces the general result (28).
} 
for the total variation along the whole renormalisation group flow $\left(C_{e f f}^{I R}=0\right.$ in a massive theory). It is easy to check that inserting in this formula Eq. (16) with (27) as the only non-zero contribution for $n>0$ gives the expected results 1 for the free boson and $1 / 2$ for the free fermion. One can also check (in the free as in the interacting case) that the approximation of the r.h.s. of (30) through the first low temperature terms (19) produces a poor result, indicating that the convergence to the intermediate and high temperature regimes is not very rapid. This fact can be contrasted with the impressively fast convergence of the form factor expansion (as a function of the distance) for the correlation functions in integrable field theory at zero temperature. This different behaviour is hardly surprising in view of the different nature of the two expansions.

Since the distribution (17) becomes singular as $R \rightarrow 0$ in the bosonic case but not in the fermionic case, the high temperature behaviour is very sensitive to the statistics. For the quadratic operators in the free cases one has

$$
\left[\langle\Theta\rangle_{R}-\langle\Theta\rangle_{R=\infty}\right]_{\text {free }}=2 M^{2} \sum_{k=1}^{\infty} S^{k-1} K_{0}(k r) \simeq\left\{\begin{aligned}
\pi M^{2} / r, & S=1 \\
-M^{2} \ln r, & S=-1
\end{aligned}\right.
$$

For the vertex operators $V_{a}=e^{a \varphi}$ with scaling dimension $X_{a}=-a^{2} / 4 \pi$, in the free theory one has $\mathcal{F}_{2 n}^{V_{a}}=a^{2 n} / 2^{n}$, so that (16) gives

$$
\left\langle e^{a \varphi}\right\rangle_{R}^{\text {free }} \simeq e^{a^{2} / 4 r}, \quad R \rightarrow 0 .
$$

In the bosonic case $S(0)=1$, it follows from the $1 / r$ behaviour of the function (17) that the $n$-particle contribution to $\langle\Phi\rangle_{R}$ behaves as $1 / r^{n}$ at high temperature. Up to unlikely cancellations of infinities, this means that the integral in the sum rule (30) diverges if $\langle\Theta\rangle_{R}$ receives contributions with $n>1$, namely if the theory is interacting. Therefore, one is led to the conclusion that in two dimensions the bosonic statistics applies only to the free case $S(\theta)=1$. An illustration of this fact is given by the sinh-Gordon model, namely the theory of a scalar field self-interacting trough the potential $\mu \cosh g \varphi$. Its exact scattering amplitude $S(\theta)=(\sinh \theta-i \sin \pi B) /(\sinh \theta+i \sin \pi B)$, with $B=g^{2} /\left(8 \pi+g^{2}\right)$, shows that $S(0)$ becomes -1 as soon as $g$ is taken different from zero. One can expect that the one-point function $\left\langle e^{a \varphi}\right\rangle_{R}$ will behave in the high temperature limit as a power law with an exponent that diverges as $g \rightarrow 0$ in order to reproduce the essential singularity (32).

We said that the operator $\Phi(x)$ entering (\$) has to be local with respect to the excitations of the zero temperature theory. We now clarify the origin of this requirement. The

\footnotetext{
${ }^{3}$ See Ref. 11] for an example of the troubles one encounters when looking for a counterexample.
} 
general situation can be illustrated through the example of the Ising field theory defined at $R=\infty$ by the action

$$
\mathcal{A}=A_{C F T}+\tau \int d^{2} x \varepsilon(x)+h \int d^{2} x \sigma(x),
$$

describing the perturbation of the Ising conformal point by the two relevant operators of the theory, the energy $\varepsilon(x)$ and the spin $\sigma(x)$.

Consider first the case of vanishing magnetic field, $h=0$, in which (33) describes a disordered phase $(\tau>0)$ and an ordered phase $(\tau<0)$ dual to each other and both corresponding to a free fermionic theory $(S=-1)$. What changes in the scattering description of the two phases is the nature of the particles, which are ordinary excitations over the unique vacuum at $\tau>0$, and kinks interpolating between the two degenerate vacua of the spontaneously broken phase at $\tau<0$. The perturbing operator $\varepsilon$ is proportional to the trace of the stress-energy tensor $(\Theta \sim \tau \varepsilon)$ and is local with respect to both kind of excitations. Its finite temperature one-point function is given by (31). The form factors of the spin operator are known in both phases [12, 13. At $\tau>0$, they are non-zero only on states with an odd number of particles so that $\langle\sigma\rangle_{R}$ vanishes, as it should by spin reversal symmetry. In the broken symmetry phase the spin couples to the states with an even number of kinks, and one could expect a non-trivial result from Eq. (16). The spin, however, is not local with respect to the kinks, and the residue (11) on the annihilation poles gets modified by a constant phase factor (a minus sign in this specific case) multiplying the product of scattering amplitudes [13]. A similar modification is induced in the factor (18) which no longer cancels the annihilation poles of the matrix elements (12), so that the expression (16) becomes badly divergent for $R<\infty$. The conclusion is that $\langle\sigma\rangle_{R}$ is not defined at $\tau<0$. Since a finite value of $\langle\sigma\rangle_{R}$ would imply the existence of spontaneous symmetry breaking on the cylinder, this result illustrates the absence of phase transitions in systems with a single infinite dimension.

The same mechanism applies to the generic case. In two (infinite) dimensions, only a discrete symmetry can be broken spontaneously. The elementary excitations in the broken phase are kinks interpolating among the discrete vacua. The order parameter is non-local with respect to them, and its expectation values on the asympotic states are incurably divergent.

Let us conclude this discussion of the Ising field theory at finite temperature by considering the other integrable direction of the action (33), namely the case $h \neq 0, \tau=0$. The spectrum of the theory [14] contains now three particles below the lowest two-particle 
threshold with masses $M_{1}$,

$$
\begin{aligned}
& M_{2}=(1.6180339887 . .) M_{1}, \\
& M_{3}=(1.9890437907 . .) M_{1} .
\end{aligned}
$$

Hence, the first terms of the low-temperature expansion for the one-point functions are

$$
\frac{\langle\Phi\rangle_{R}}{\langle\Phi\rangle_{R=\infty}}=1+\frac{1}{\pi} \sum_{i=1}^{3} A_{i}^{\Phi} K_{0}\left(M_{i} R\right)+O\left(e^{-2 M_{1} R}\right),
$$

where the ratios

$$
A_{i}^{\Phi}=\frac{\left\langle 0|\Phi(0)| a_{i}(i \pi), a_{i}(0)\right\rangle}{\langle\Phi\rangle_{R=\infty}},
$$

are universal and can be extracted from the work of Refs. [15, 16] on the form factors of the Ising model in a magnetic field. We give their values in Table 1.

\begin{tabular}{|c||c|c|}
\hline$\Phi$ & $\sigma$ & $\varepsilon$ \\
\hline$A_{1}^{\Phi}$ & $-8.0999744 .$. & $-17.893304 .$. \\
$A_{2}^{\Phi}$ & $-21.206008 .$. & $-24.946727 .$. \\
$A_{3}^{\Phi}$ & $-32.045891 .$. & $-53.679951 .$. \\
\hline
\end{tabular}

Table 1. The universal amplitudes entering the expansion (34) for the Ising model in a magnetic field.

Notice that in this case the trace of the stress-energy tensor is proportional to the spin operator. Hence, the amplitudes $A_{i}^{\sigma}$ are $2 \pi M_{i}^{2}$ divided by $\langle\sigma\rangle_{R=\infty}$. The latter quantity is also known from the TBA [17]. The amplitudes $A_{i}^{\varepsilon}$, instead, can only be obtained through a form factor computation [16] in which the 'cluster' property explained in Ref. [18 plays an essential role.

In conclusion, the form factor approach provides a systematic and explicit low temperature expansion for the one-point functions in integrable field theory at finite temperature. Form factors have already been computed for many integrable theories and it is highly desirable to have numerical data to compare with the theoretical predictions. It also seems natural to expect that the study of the two-point functions should be approachable along the same lines with the goal of obtaining a low temperature, large distance expansion. This problem is beyond the scope of this note and will be considered elsewhere.

Acknowledgments: I thank G. Mussardo for interesting discussions.

\footnotetext{
${ }^{4}$ Here $a_{i}(\theta)$ denotes a particle with mass $M_{i}$ and rapidity $\theta$.
} 


\section{References}

[1] A. LeClair, F. Lesage, S. Sachdev and H. Saleur, Nucl. Phys. B 482 (1996), 579.

[2] A. LeClair and G. Mussardo, Nucl. Phys. B 552 (1999), 624.

[3] H. Saleur, Nucl. Phys. B 567 (2000), 602.

[4] S. Lukyanov, Finite temperature expectation values of local fields in the sinh-Gordon model, hep-th/0005027.

[5] A.B. Zamolodchikov and Al.B. Zamolodchikov, Ann. Phys. 120 (1979), 253.

[6] M. Karowski and P. Weisz, Nucl. Phys. B 139 (1978), 455.

[7] F.A. Smirnov, Form Factors in Completely Integrable Models of Quantum Field Theories (World Scientific) 1992.

[8] G. Delfino, G. Mussardo and P. Simonetti, Nucl. Phys. B 473 (1996), 469.

[9] Al.B. Zamolodchikov, Nucl. Phys. B 342 (1990), 695.

[10] J.L. Cardy, J. Phys. A 16 (1984), L385.

[11] G. Mussardo and P. Simon, Nucl. Phys. B 578 (2000), 527.

[12] B. Berg, M. Karowski and P. Weisz, Phys. Rev. D 19 (1979), 2477.

[13] V.P. Yurov and Al.B. Zamolodchikov, Int. J. Mod. Phys. A6 (1991), 3419.

[14] A.B. Zamolodchikov, Adv. Stud. Pure Math. 19 (1989), 641.

[15] G. Delfino and G. Mussardo, Nucl. Phys. B 455 (1995), 724.

[16] G. Delfino and P. Simonetti, Phys. Lett. B 383 (1996), 450.

[17] V.A. Fateev, Phys. Lett. B 324 (1994), 45.

[18] G. Delfino, P. Simonetti and J.L. Cardy, Phys. Lett. B 387 (1996), 327. 